\title{
ESTUDO RETROSPECTIVO DA LEPTOSPIROSE CANINA EM HOSPITAL VETERINÁRIO ESCOLA NO MUNICÍPIO DE LONDRINA, PR
}

\author{
Amanda Bertão-Santos ${ }^{1}$ \\ Letícia Santos Balbino ${ }^{2}$ \\ Fernanda Pinto-Ferreira ${ }^{3}$ \\ Eloiza Teles Caldart ${ }^{4}$ \\ Marcelle Mareze ${ }^{5}$ \\ Leonardo Oliveira Lopes Silva ${ }^{6}$ \\ Elena Castellani ${ }^{7}$ \\ Ana Carolina Miura ${ }^{8}$ \\ Lucienne Garcia Pretto Giordano9 \\ Italmar Teodorico Navarro ${ }^{10}$
}

BERTÃO-SANTOS, A.; BALBINO, L. S.; PINTO-FERREIRA, F.; CALDART, E. T.; MAREZE, M.; SILVA, L. O. L.; CASTELlANI, E.; MIURA, A. C.; GIORDANO, L. G. P.; NAVARRO, I. T. Estudo retrospectivo da leptospirose canina em hospital veterinário escola no município de Londrina, Pr. Arquivos de Ciências Veterinárias e Zoologia da UNIPAR, Umuarama, v. 24, n. 2cont., e2409, 2021.

RESUMO: O objetivo do trabalho foi relatar a ocorrência de casos de cães com Leptospira spp. no Hospital Veterinário de Londrina. Foram avaliados 5.454 prontuários, durante os meses de janeiro a dezembro de 2016, e selecionados os que apresentaram solicitação de exame para Lepstopira por meio da técnica de campo escuro (CE). Nos positivos pela técnica CE dados sobre o sexo, sinais clínicos, endereço, data do atendimento e resultado na SAM foram coletados. A análise dos dados foi realizada pelo programa Epilnfo (7.2.3.1), dos 5.454 prontuários nos quais foi solicitado exame CE em 7,06\% (385/5.454), e nesses, 13,76\% (53/385) foi identificada a presença da espiroqueta. Dos animais positivos na técnica CE, 20,75\% (11/53) foram reagentes na SAM. O sorovar Canicola foi o mais frequente em 90,91\% (10/11) dos animais, apresentando títulos variados de 100 a 12.800. Para o sorovar Pomona 9,09\% (1/11) dos animais apresentaram títulação de 100. Os sinais clínicos mais observados foram apatia em 58,49\% (31/53), azotemia em 49,06\% (26/53) e êmese em 49,06\% (26/53) dos casos. Quanto ao sexo, os machos constituíram $71,70 \%$ dos casos e as fêmeas $28,30 \%(\mathrm{p}=0,0015)$. Os resultados demonstraram a presença de Leptospira spp. entre os animais atendidos no hospital veterinário de Universidade Estadual de Londrina e reforçam a importância do sorovar Canicola entre os cães, visto que esse foi o mais frequente entre os sorovares e que apresentou os maiores títulos.

PALAVRAS-CHAVE: Exame direto. Leptospira spp. Sorologia.

\section{RETROSPECTIVE STUDY OF CANINE LEPTOSPIROSIS IN A VETERINARY SCHOOL HOSPITAL IN THE MUNICIPALITY OF LONDRINA, PR}

\footnotetext{
ABSTRACT: The purpose of this work is to report the occurrence of cases of dogs with Leptospira spp. at the Veterinary Hospital of Londrina. A total of 5,454 medical records were evaluated from January to December 2016, and data obtained from the examination for Lepstopira using the dark field technique (DF) were selected. In cases which were positive by the DF technique, data on gender, clinical signs, address, care data, and SAM results were collected. Data analysis was performed using the EpiInfo program (7.2.3.1). From the total 5,454 medical records in which DF examination was requested, in $7.06 \%$ (385/5.454), and from those, 13.76\% (53/385) were positive for Leptospira. From the animals positive in the DF technique, $20.75 \%(11 / 53)$ were reactive in SAM. The Canicola serovar was the most prevalent, being present in $90.91 \%(10 / 11)$ of the animals, with titers ranging from 100 to 12,800. For the Pomona serovar, $9.09 \%(1 / 11)$ of the animals presented a title of

DOI: 10.25110 /arqvet.v24i2cont.2021.8612

${ }^{1}$ Acadêmica do curso de Medicina Veterinária, Universidade Estadual de Londrina. amandabertao9@gmail.com

${ }^{2}$ Acadêmica do curso de Medicina Veterinária, Universidade Estadual de Londrina. leticia.balbino@uel.br

${ }^{3}$ Médica Veterinária. Doutora em Ciência Animal (UEL). Docente de Parasitologia Veterinária na Universidade Estadual de Londrina.fernandaferreira@uel.br

${ }^{4}$ Médica Veterinária. Doutora em Ciência Animal (UEL). Docente de Parasitologia Veterinária na Universidade Estadual de Londrina. eloizacaldart@uel.br

${ }^{5}$ Médica Veterinária. Doutorado em Ciência Animal (UEL).marcelle_mareze@hotmail.com

${ }^{6}$ Acadêmico do curso de Medicina Veterinária, Universidade Estadual de Londrina. leonardolsilva07@gmail.com

${ }^{7}$ Acadêmica do curso de Medicina Veterinária, Universidade Estadual de Londrina. elena.castellani@uel.br

${ }^{8}$ Médica Veterinária. Doutora em Ciência Animal (UEL). anacarolina.miura@gmail.com

${ }^{9}$ Médica Veterinária. Doutora em Ciência Animal (UEL). Docente de Microbiologia Veterinária na Universidade Estadual de Londrina. lgiordano@uel.br

${ }^{10}$ Médico Veterinário. Doutor em Epidemiologia Experimental Aplicada ás Zoonoses pela Universidade de São Paulo. Docente de Zoonoses na Universidade

Estadual de Londrina. italmar@uel.br
} 
100. The most frequent clinical signs observed were apathy, in 58.49\% (31/53); azotemia in 49.06\% (26/53); and emesis in $49.06 \%(26 / 53)$ of the cases. As for gender, male animals constituted $71.70 \%$ of the cases and female $28.30 \%(p=0.0015)$. The results demonstrated the presence of Leptospira spp. among animals treated at the Veterinary Hospital of the State University of Londrina which reinforces the importance of the Canicola serovar among dogs, since it was the serovar with the highest prevalence and highest titer found.

KEYWORDS: Direct exam. Leptospira spp. Serology.

\section{ESTUDIO RETROSPECTIVO DE LEPTOSPIROSIS CANINA EN UN HOSPITAL VETERINARIO ESCOLAR DEL MUNICIPIO DE LONDRINA, PR}

RESUMEN: El objetivo de este trabajo fue reportar la ocurrencia de casos de perros con Leptospira spp. en el Hospital Veterinario de Londrina. Se evaluaron un total de 5.454 historias clínicas de enero a diciembre de 2016, y se seleccionó los que presentaron solicitud de examen de Lepstopira mediante la técnica de campo oscuro (CE). En los positivos por la técnica de CE se recogieron datos de sexo, signos clínicos, domicilio, fecha de atención y resultado en la SAM. El análisis de los datos se realizó mediante el programa EpiInfo (7.2.3.1), de las 5.454 historias clínicas en las que se solicitó examen CE en el 7,06\% (385/5.454), y en estas, el 13,76\% (53/385) se identificaron la de espiroqueta. De los animales positivos en la técnica CE, el 20,75\% (11/53) fueron reactivos en la SAM. El serovar Canicola fue el más frecuente en el 90,91\% (10/11) de los animales, con títulos que oscilaron entre 100 y 12.800. Para el serovar Pomona, el 9.09\% (1/11) de los animales tuvo un título de 100 . Los signos clínicos más observados fueron apatía en 58.49\% (31/53), azotemia en $49.06 \%(26 / 53)$ y emesis en $49.06 \%(26 / 53)$ de los casos. Cuanto al sexo, los machos constituyeron el 71,70\% de los casos y las hembras el 28,30\% (p = 0,0015). Los resultados demostraron la presencia de Leptospira spp. entre los animales tratados en el Hospital Veterinario de la Universidad Estatal de Londrina y refuerzan la importancia del serovar Canicola entre los perros, ya que este fue el más frecuente entre los serovares y tuvo los títulos más altos.

PALABRAS CLAVE: Examen directo. Leptospira spp. Serología.

\section{Introdução}

A leptospirose é uma doença zoonótica de distribuição global, causada por bactérias do gênero Leptospira spp., as quais são classificadas em mais de 250 sorovares, capazes de infectar humanos e animais (LEVETT, 2001). Em humanos, essa doença está associada a altos níveis de pluviosidade e temperatura, assim como, a condições sociais e sanitárias precárias. Esses fatores favorecem o estabelecimento dos roedores, o acúmulo de água e, consequentemente, a transmissão e o aumento da incidência; além disso, explicam o fato da doença ser endêmica em países tropicais (ADLER; DE LA PEÑA MOCTEZUMA, 2010; JORGE et al., 2017).

Nos animais a infecção pode ser assintomática ou apresentar amplo espectro de sinais clínicos. Todos os mamíferos selvagens ou domésticos podem ser reservatórios da Leptospira (ANDERSEN-RANBERG; PIPPER; JENSEN, 2016; KO; GOARANT; PICARDEAU, 2009). Os cães são hospedeiros de manutenção do sorovar Canicola, neles a bactéria induz doença renal crônica, e o hospedeiro mesmo que assintomático pode eliminar a bactéria na urina (CORTEZ et al., 2020; MUÑOZ-ZANZI et al., 2014).

A infecção pode ocorrer quando há contato da bactéria com micro lesões na pele, contato com a mucosa, conjuntiva ou inalação via aerossol (ZAIDI et al., 2018). A transmissão da bactéria geralmente ocorre indiretamente pelo contato com água ou solo contaminados; sabe-se que a Leptospira não resiste a ambientes secos e com pH ácido (TRUEBA et al., 2004). A transmissão direta se dá pelo contato com a urina de animais infectados (PICARDEAU, 2013).

Para o diagnóstico, testes moleculares estão ganhando mais espaço, pois são capazes de detectar precocemente as infecções em animais assintomáticos e sintomáticos (MIOTTO et al., 2017; REAGAN; SYKES, 2019). No entanto, testes sorológicos, como a soroaglutinação microscópica (SAM), ainda são os mais utilizados devido à facilidade e baixo custo para a realização, embora apresentem limitações (FAINE et al., 1999). A visualização em microscopia de campo escuro (CE), também é uma técnica muito aplicada, que possibilita a observação da espiroqueta, mas deve ser utilizada com parcimônia, já que se trata de um diagnóstico morfológico (FAINE et al., 1999).

O município de Londrina $\left(23^{\circ} 18^{\prime} 36^{\prime \prime} \mathrm{S}\right.$ e $\left.51^{\circ} 09^{\prime} 46^{\prime \prime} \mathrm{O}\right)$, Paraná, Sul do Brasil, apresenta um índice de desenvolvimento humano (IDH) de 0.841, uma população total estimada para 2019 de 569.733 habitantes, sendo que 97,0\% vivem em área urbana (IBGE, 2019). Dos 399 municípios do estado do Paraná, Londrina está entre os cinco com maior número de casos de leptospirose em humanos registrados, com 67,39\% deles em área urbana (BRASIL, 2019).

O presente estudo teve por objetivo relatar a ocorrência de casos de cães com leptospirose atendidos em um Hospital Veterinário Escola, no município de Londrina no ano de 2016.

\section{Materiais e Métodos}

Para o desenvolvimento do presente trabalho foram avaliados os prontuários de todos os animais atendidos de janeiro a dezembro de 2016, no Hospital Veterinário da Universidade Estadual de Londrina. Na primeira seleção, foram classificados os prontuários de cães investigados para leptospirose. Em seguida foram selecionados os prontuários 
com resultados positivos na microscopia de campo escuro (CE) (FAINE, 1999), desses foram extraídos dados como: sexo, sinais clínicos, endereço, data do atendimento e resultado da SAM, quando realizada. Os dados dos animais obtidos nos prontuários, bem como os resultados dos exames foram digitados no programa EpiInfo (7.2.3.1), o qual foi utilizado para a estatística descritiva.

\section{Resultados}

Foi avaliado um total de 5.454 prontuários, em $7,06 \%(385 / 5.454)$ deles, foi solicitado o teste de CE, $13,76 \%$ $(53 / 385)$ desses foram considerados positivos. Dentre os animais positivos, $71,70 \%$ eram machos e $28,30 \%$ eram fêmeas ( $\mathrm{p}=0,0015)$. Os sinais clínicos reportados foram: apatia em 58,49\% (31/53), azotemia em 49,06\% (26/53), êmese em $49,06 \%$ (26/53), anorexia em 40,38\% (21/53), anemia em $30,91 \%$ (17/53), desidratação em 25,45\% (14/53), diarreia em $23,64 \%$ (13/53), dor abdominal em 14,55\% (8/53), icterícia em $9,09 \%(5 / 53)$, necrose de língua em 3,64\% (2/53) e urina marrom em $1,82 \%(1 / 53)$.

Dos animais positivos no CE, 20,75\% (11/53) foram reagentes na SAM, dentre esses, 9,09\% (1/11) para o sorovar Pomona apresentando o título de 100 , e 90,91\% (10/11) para o sorovar Canicola apresentando títulos variados. A tabela 1 apresenta as características, o sorovar e o título dos animais positivos na $\mathrm{CE}$ e reagentes para a SAM. A distribuição mensal dos casos de leptospirose canina está apresentada na Figura 1 e a distribuição espacial dos casos está apresentada na Figura 2.

Tabela 1: Dados dos animais positivos no CE e SAM, principal sorovar envolvido, título, gênero, espécie, raça e cidade dos animais reagentes.

\begin{tabular}{|c|c|c|c|c|c|c|c|}
\hline ANIMAL & SOROVAR & TITULO & GÊNERO & EPÉCIE & RAÇA & CIDADE & ESTADO \\
\hline 1 & Canicola & 12800 & macho & canina & SRD & Cambé & PR \\
\hline 2 & Canicola & 800 & macho & canina & SRD & Londrina & PR \\
\hline 3 & Canicola & 100 & macho & canina & Labrador & Londrina & PR \\
\hline 4 & Pomona & 100 & macho & canina & SRD & Ibiporã & PR \\
\hline 5 & Canicola & 1600 & fêmea & canina & SRD & Cambé & PR \\
\hline 6 & Canicola & 1600 & macho & canina & Pittbull & Londrina & PR \\
\hline 7 & Canicola & 1600 & macho & canina & SRD & Londrina & PR \\
\hline 8 & Canicola & 800 & macho & canina & Pinscher & Rolândia & PR \\
\hline 9 & Canicola & 3200 & macho & canina & Lhasa apso & Londrina & PR \\
\hline 10 & Canicola & 800 & macho & canina & Shitzu & Londrina & PR \\
\hline 11 & Canicola & 100 & macho & canina & SRD & Londrina & PR \\
\hline
\end{tabular}

Figura 1: Distribuição mensal dos casos de leptospirose canina diagnosticados por microscopia em campo escuro (CE) no Hospital Veterinário da UEL de janeiro a dezembro de 2016 no município de Londrina.
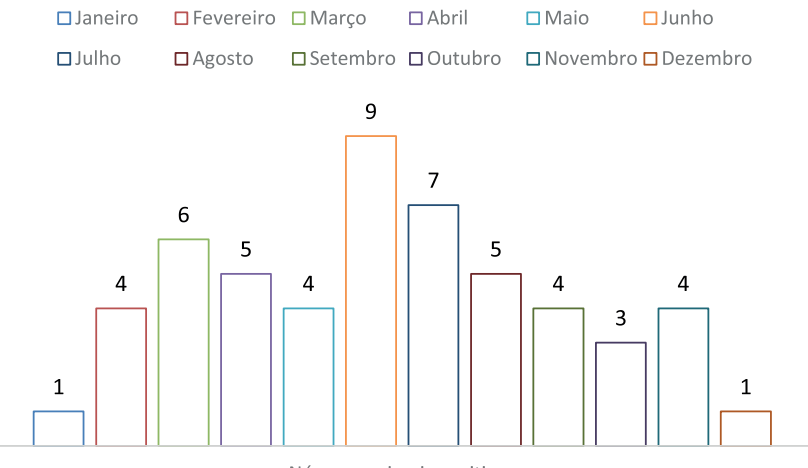

Número animais positivos
Figura 2: Distribuição espacial dos casos de leptospirose canina diagnosticados no Hospital Veterinário da UEL de janeiro a dezembro de 2016, em municípios da região Norte do Paraná

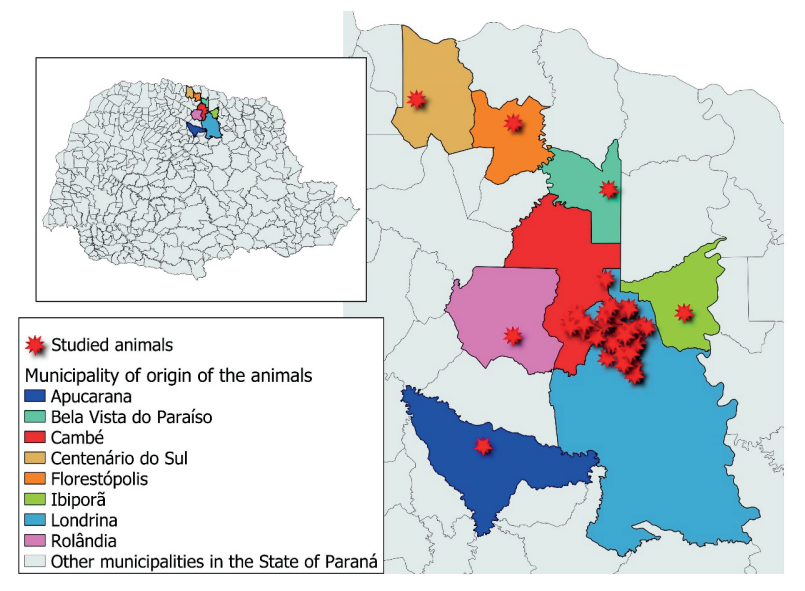

\section{Discussão}

O CE apresenta limitações, pois deve ser realizado no máximo 30 minutos após a colheita da urina, já que o diagnóstico 
é baseado na análise da morfologia e motilidade das leptospiras e depende de observador experiente. Ainda, como a eliminação da bactéria pela urina é intermitente, pode ser necessária mais de uma coleta. Sendo assim, não é recomendada a técnica $\mathrm{CE}$ como único teste a ser solicitado para o diagnóstico laboratorial de leptospirose. Já a técnica de diagnóstico SAM é a eleita como padrão ouro pela Organização Mundial de Saúde (OMS), no entanto, devido as particularidade e limitações, deve ser utilizada com parcimônia, aliada às informações a respeito do histórico, sintomatologia clínica do animal e exame direto, como a microscopia de Campo Escuro, possibilitando uma maior confiabilidade nos resultados (BRASIL, 2014; FAINE, 1982). A SAM é um teste que detecta anticorpos contra os sorovares de Leptospira spp. Os anticorpos aparecem entre os seis e 12 primeiros dias pós-infecção, apresentando títulos máximos entre a terceira e quarta semana seguinte, com o decorrer do tempo, títulos decrescem a níveis basais, e podem persistir por meses ou anos. Torna-se necessário o conhecimento prévio sobre o histórico e sinais clínicos do animal, para que se diferenciem os títulos de anticorpos quanto às infecções ativas, passadas, ou títulos vacinais (SIMÕES et al., 2016).

Neste estudo, os animais considerados positivos foram avaliados nas técnicas SAM, CE e apresentaram sinais clínicos compatíveis com aqueles frequentemente encontrados na leptospirose. A maior frequência do sorovar Canicola pode ser justificada pela adaptação epidemiológica deste sorovar aos cães, que atuam como seu principal hospedeiro de manutenção (SYKES et al., 2011). Animais infectados podem desenvolver quadro agudo, crônico ou subclínico. Nos cães os sinais estão principalmente ligados à lesão renal, esses animais podem eliminar a bactéria por meio da urina por longos períodos, possibilitando a infecção do homem e de outros animais (SILVESTRINI; HEINEMANN; DE CASTRO, 2019; SYKES et al., 2011). O sorovar Canicola também foi o que apresentou os maiores títulos, sabe-se que a situação vacinal do animal deve ser levada em conta, pois pode interferir nos resultados (BARR et al., 2005; KNÖPFLER et al., 2017; SYKES et al., 2011). A patogenicidade e os sinais clínicos apresentados pelos animais variam e são influenciados pela idade do hospedeiro, resposta imune, virulência da cepa infectante e carga bacteriana. Envolvem, muitas vezes, sinais inespecíficos como: anorexia, vômito, apatia e febre, que foram observados no presente estudo. Na fase aguda, pode ocorrer lesão hepática e renal, assim, os sinais como a azotemia, segundo sinal clínico mais relatado $(49,06 \%)$, icterícia e anemia, podem ter relação com as alterações causadas pela espiroqueta nesses órgãos (SCHULLER et al., 2015; SYKES et al., 2011). O acesso à rua e o contato entre cães portadores favorece a transmissão da bactéria (BENITEZ et al., 2012; FERNANDES et al., 2018; MASCOLLI et al., 2016). Observou-se predomínio de infecção entre cães machos. Acredita-se que o hábito territorialista de farejar a urina de outros animais, assim como comportamentos sexuais ou de reconhecimento possam contribuir para a infecção dos machos (MODOLO et al., 2006). Dentre os animais atendidos, a maioria residia no município de Londrina, onde se localiza o Hospital Veterinário. Os casos externos ao município evidenciam a popularização do atendimento veterinário da Universidade, referência na região norte do Estado do Paraná.

\section{Conclusão}

Os resultados demonstram a presença de Leptospira spp. entre os animais atendidos no Hospital Veterinário de Universidade Estadual de Londrina e reforçam a importância do sorovar Canicola entre os cães, visto que esse foi o mais frequente entre os sorovares e que apresentou os maiores títulos.

\section{Referências}

ADLER, B.; DE LA PEÑA MOCTEZUMA, A. Leptospira and leptospirosis. Veterinary Microbiology, v. 140, n. 3-4, p. 287-296, 2010.

ANDERSEN-RANBERG, E. U.; PIPPER, C.; JENSEN, P. M. Global patterns of Leptospira prevalence in vertebrate reservoir hosts. Journal of wildlife diseases. Journal of Wildlife Diseases, v. 52, n.3, p. 468-477, 2016.

BARR, S. C. et al. Serologic responses of dogs given a commercial vaccine against Leptospira interrogans serovar Pomona and Leptospira Kirschneri serovar Grippotyphosa. American Journal of Veterinary Research, v. 66, n. 10, p. 1780-1784, 2005.

BENITEZ, A. do N. et al. Soroepidemiology of leptospirosis in pet dogs in the urban area of the municipality of Jataizinho, Paraná. Semina: Ciências Agrárias, Londrina, v. 33, n. Suppl. 2, p. 3201-3210, 2012.

BRASIL. MINISTÉRIO DA SAÚDE. Secretaria de Vigilância em Saúde. Departamento de Vigilância das Doenças Transmissíveis. Leptospirose: diagnóstico e manejo clínico / Ministério da Saúde, Secretaria de Vigilância em Saúde. Departamento de Vigilância das Doenças Transmissíveis. - Brasília : Ministério da Saúde, 2014.

\section{BRASIL. MINISTÉRIO DA SAÚDE. Sistema de} Informação de Agravos de Notificação - Sinan Net. Disponível em: < http://tabnet.datasus.gov.br/cgi/tabcgi. exe?sinannet/cnv/leptopr.def > . Acesso em: 27 ago. 2019.

CORTEZ, A. et al. Canine leptospirosis in asymptomatic populations from the Southwest Region of São Paulo State, Brazil. Brazilian Journal of Veterinary Research and Animal Science, v. 57, n. 4, p. e167893-e167893, 2020.

FAINE S. Guidelines for the control of Leptospirosis. 1982, World Health Organization: Geneva.

FAINE, S.et al. Leptospira and leptospirosis. Melbourne: Medisci, 2ed., 1999. 
FERNANDES, A. R. F. et al. Soropositividade e fatores de risco para leptospirose, toxoplasmose e neosporose na população canina do Estado da Paraíba. Pesquisa Veterinária Brasileira, v. 38, n. 5, p. 957-966, 2018.

IBGE. População estimada: IBGE, Diretoria de Pesquisas, Coordenação de População e Indicadores Sociais, Estimativas da população residente com data de referência $1^{\circ}$ de julho de 2019.

JORGE, S. et al. Human and animal leptospirosis in Southern Brazil: A five-year retrospective study. Travel Medicine and Infectious Disease, v. 18, p. 46-52, 2017.

KNÖPFLER, S. et al. Evaluation of clinical, laboratory, imaging findings and outcome in 99 dogs with leptospirosis. Journal of Small Animal Practice, v. 58, n. 10, p. 582-588, 2017.

KO, A. I.; GOARANT, C.; PICARDEAU, M. Leptospira: The dawn of the molecular genetics era for an emerging zoonotic pathogen. Nature Reviews Microbiology, Nature Publishing Group, 2009.

\section{LEVETT, P. N. LeptospirosisClinical Microbiology}

Reviews, 2001.

MASCOLLI, R. et al. Prevalência e fatores de risco para a leptospirose e brucelose na população canina da Estância Turística de Ibiúna, São Paulo, Brasil. Arquivos do Instituto Biológico, v. 83, 2016.

MIOTTO, B. A. et al. Development and validation of a modified TaqMan based real-time PCR assay targeting the lipl32 gene for detection of pathogenic Leptospira in canine urine samples. Brazilian Journal of Microbiology, v. 49, n. 3, p. 584-590, 2017.

MODOLO, J. R. et al. Investigação soroepidemiológica de leptospirose canina na área territorial urbana de Botucatu, São Paulo, Brasil. Brazilian Journal of Veterinary Research and Animal Science, v. 43, n. 5, p. 598-604, 2006.

MUÑOZ-ZANZI, C. et al. Leptospira Contamination in Household and Environmental Water in Rural Communities in Southern Chile. International Journal of Environmental Research and Public Health, v. 11, n. 7, p. 6666-6680, 26 jun. 2014.

PICARDEAU, M. Diagnosis and epidemiology of leptospirosis. Medecine et Maladies Infectieuses, v. 43, n. 1, p. 1-9, 2013.

REAGAN, K. L.; SYKES, J. E. Diagnosis of canine leptospirosis. Veterinary Clinics: Small Animal Practice, v. 49, n. 4, p. 719-731, 2019.

SCHULLER, S. et al. European consensus statement on leptospirosis in dogs and cats. Journal of Small Animal
Practice, v. 56, n. 3, p. 159-179, 2015.

SILVESTRINI, A. R.; HEINEMANN, M. B.; DE CASTRO, A. M. M. G. Leptospirose no contexto da Saúde Única e diretrizes de vacinação. PUBVET, v. 14, p. 137, 2019.

SIMÕES, L. S. et al. Leptospirose-Revisão. PUBVET, v. 10, p. 138-146, 2016.

SYKES, J. E. et al. 2010 ACVIM small animal consensus statement on leptospirosis: diagnosis, epidemiology, treatment, and prevention. Journal of Veterinary Internal Medicine, v. 25, n. 1, p. 1-13, 2011.

TRUEBA, G. et al. Cell aggregation: A mechanism of pathogenic Leptospira to survive in fresh water. International Microbiology, v. 7, n. 1, p. 35-40, 2004.

ZAIDI, S. et al. Urinary shedding of pathogenic leptospira in stray dogs and cats, Algiers: A prospective study. PLoS ONE, v.13, n.5, mai. 2018. 